\title{
A computational study of the interaction noise from a small axial-flow fan
}

\author{
H. Z. Lu \\ Department of Mechanical Engineering, The Hong Kong Polytechnic University, Kowloon, \\ Hong Kong, China \\ Lixi Huang ${ }^{\text {a) }}$ \\ Department of Mechanical Engineering, The University of Hong Kong, Pofulam Road, Hong Kong, \\ China \\ R. M. C. So and J. Wang \\ Department of Mechanical Engineering, The Hong Kong Polytechnic University, Kowloon, \\ Hong Kong, China
}

(Received 27 June 2006; revised 15 May 2007; accepted 1 July 2007)

\begin{abstract}
Small axial-flow fans used for computer cooling and many other appliances feature a rotor driven by a downstream motor held by several cylindrical struts. This study focuses on the aerodynamic mechanism of rotor-strut interaction for an isolated fan. The three-dimensional, unsteady flow field is calculated using FLUENT ${ }^{\circledR}$, and the sound radiation predicted by acoustic analogy is compared with measurement data. Striking differences are found between the pressure oscillations in various parts of the structural surfaces during an interaction event. The suction surface of the blade experiences a sudden increase in pressure when the blade trailing edge sweeps past a strut, while the process of pressure decrease on the pressure side of the blade is rather gradual during the interaction. The contribution of the latter towards the total thrust force on the structure is cancelled out significantly by that on the strut. In terms of the acoustic contributions from the rotor and strut, the upstream rotor dominates and this feature differs from the usual rotor-stator interaction acoustics in which the downstream part is responsible for most of the noise. It is therefore argued that the dominant interaction mechanism is potential flow in nature. (C) 2007 Acoustical Society of America. [DOI: 10.1121/1.2764474]
\end{abstract}

PACS number(s): 43.28.Ra, 43.50.Nm, 43.50.Ed [GCL] Pages: 1404-1415

\section{INTRODUCTION}

This study is motivated by the aeroacoustics of computer cooling fan. The problem of cooling fan noise is receiving increasing attention as more CPU chips are condensed in a smaller space demanding intense ventilation. Should the ventilation capacity double in the near future, the noise radiation would be increased by about $18 \mathrm{~dB}$ based on a simplistic 6th power law estimate. To prevent this problem from becoming a stumbling block of computer hardware development, a much quieter fan design has to be developed. A typical fan noise spectrum consists of two components: a broadband noise caused mainly by turbulent flow features, and tones at the blade passing frequency and its harmonics. To human ears, the emergence of tones is much more annoying than the broadband noise of equal sound energy level. Tones are mainly generated by the deterministic, unsteady loading on rotor blades and motor struts. For a cooling fan consisting of an axial-flow rotor and a set of downstream motor struts, as shown in Fig. 1(a), there are two types of interactions. The first type of interaction is between eddies in the distorted inlet flow and the rotating blades. The inlet flow is typically nonuniform in a computer chassis. Furthermore,

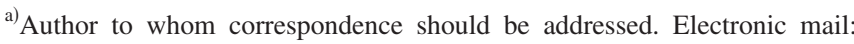
lixi.huang@hku.hk
}

eddies in a turbulent inlet flow may get elongated in a contracting inlet stream tube and get chopped by blades giving rise to tone noise, cf. (Trunzo et al., 1981), (Majumdar and Peake, 1998), (Chiu et al., 1989), and (Washburn and Lauchle, 1998). The second type of interaction is between the rotor wake and the struts in close proximity.

The first interaction mechanism is often the more powerful sound radiator than the second, but, in principle, it is possible to reduce its strength by careful geometric design for both the fan inlet and the objects surrounding the cooling fan. However, the second mechanism is unavoidable, and it is the topic of the present investigation. Most existing knowledge on this aspect of fan aeroacoustics is derived from studies of rotor-stator, or inlet guide-vane-rotor interaction in ducted turbofans (Blake, 1986), where the downstream set of blades experience most of the unsteady loading, hence sound radiation, from the interaction event. The unsteady loading is derived from both potential field interactions and viscous wake impingement. For such interactions, semiempirical models (Kemp and Sears, 1953, 1955) still serve as a good starting point. Potential field interaction dominates when the gap is less than one half of a blade chord or so (Lowson, 1965 , 1970). The potential flow interaction is particularly strong when the percentage of the flow blockage by the downstream struts is large. For the cooling fan studied in this work, it is around $15 \%$. The reason why the downstream set 


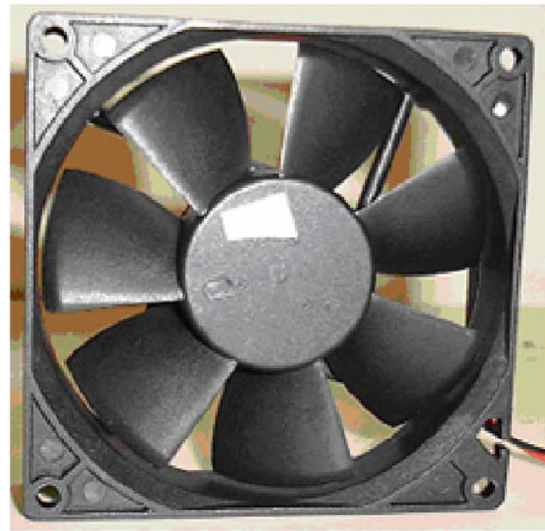

(a)

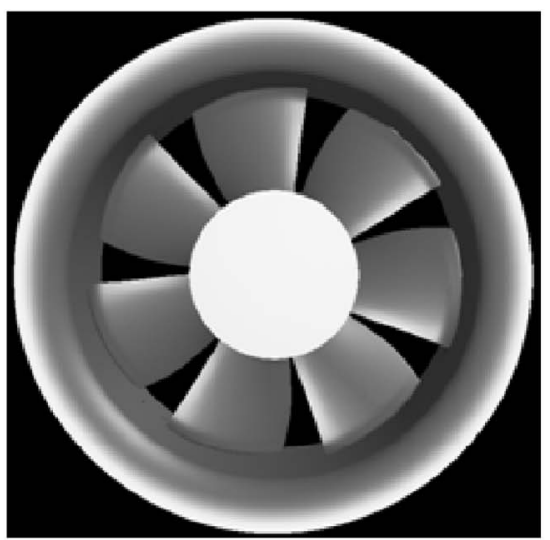

(b)

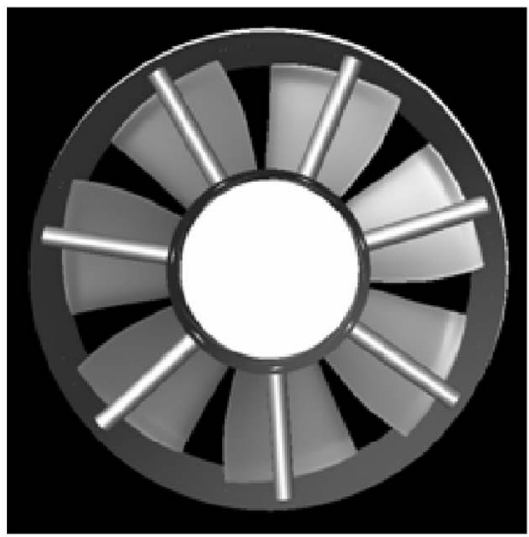

(c)

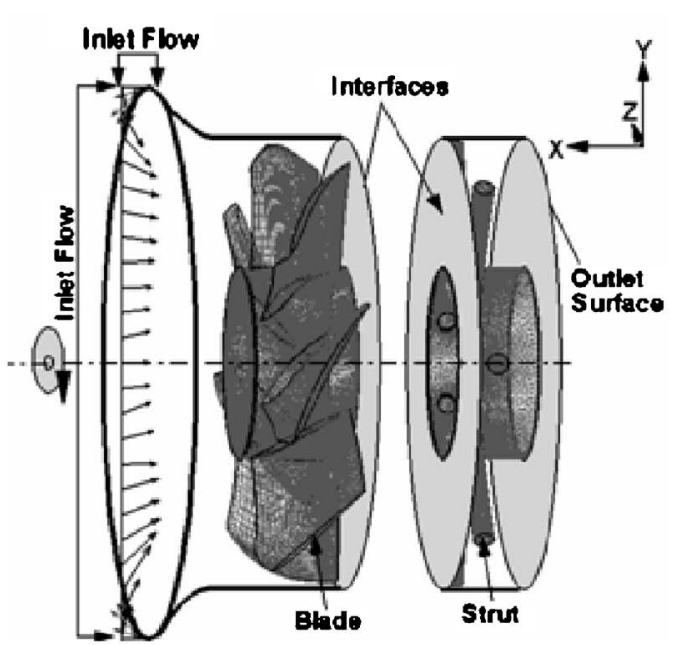

(d)

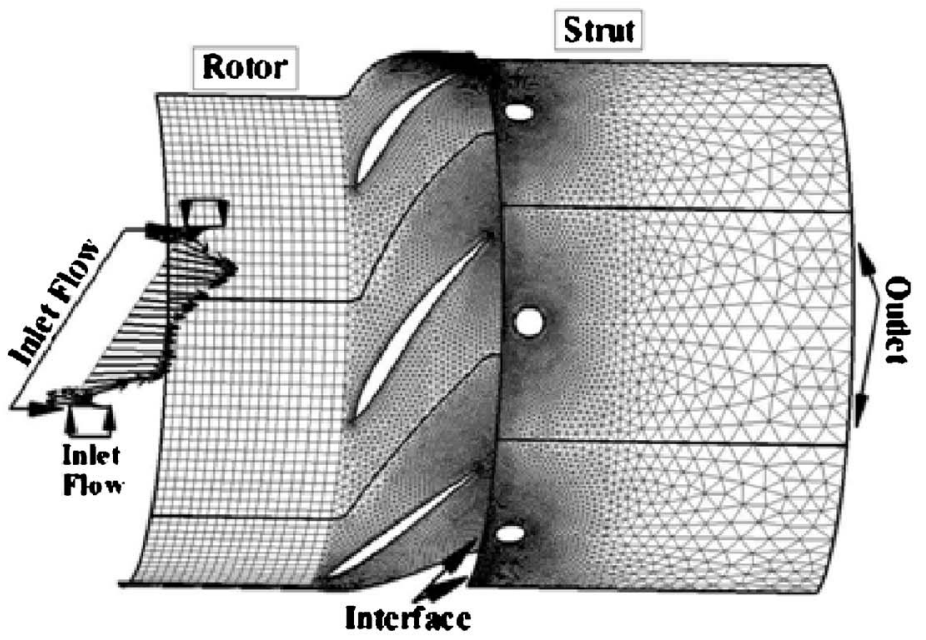

(e)

FIG. 1. The computational and experimental configuration. (a) Photo of the real cooling fan on which the computational model is based. (b) Front view of the model fan with circular bellmouth. (c) Back view of the model fan showing seven radial struts. (d) Computational model with extended inlet and exit ducts. (e) Mesh on a cylindrical cross section at $70 \%$ of the radial span.

of blades, rotor or stator, as the case may be, bears more fluctuating loading is due to the aerodynamic sensitivity of the blade leading edge. When the downstream set of blades is cylindrical struts, the forces generated might not be as large. It could be even less than that on the upstream rotor blades since, after all, most rotor blades are designed to generate lift. It is anticipated that the forces on both rotor blades and the downstream struts could be equally important. Apart from the interaction between different blade rows, interaction between the tip leakage vortex with the neighboring blades of the same blade row can also be important in terms of both broadband and tonal noise (Fukano et al., 1986; Cudina, 1992; Kameier and Neise, 1997).

In terms of the unsteady fluid dynamics during the interblade-row interaction, there have been quite a few papers on the topic in the turbomachinery community, e.g. (Hodson, 1985), (Mayle and Dullenkopf, 1989), (Mailach and Volgeler, 2004a), which are mainly focused on the viscous effects of the upstream wake on the downstream blade. Studies on the reaction of the downstream blades on the upstream blade rows due to the potential flow effect are rare (Mailach and Volgeler, 2004b), apparently due to its much weaker strength when compared with the viscous effects from the upstream blades on the downstream blades, with the exception of Mailach et al., (2003) in which the guide vane wake from the upstream is rather thin. To the best of the authors' knowledge, the interaction between an upstream blade and a downstream bluff body has not been studied apart from the preliminary effort made by the second author and his colleagues (Huang et al., 2003) using a coarse, quasi-orthogonal mesh which was not adaptable enough to cope with the complex geometry. In this study, FLUENT® (version 6.3) is used to simulate the three-dimensional (3D), unsteady flow through a complete but isolated cooling fan. Admittedly, the commercial software package may not resolve all details of the unsteady flow field that could be of potential interest to acoustics, especially for such a complicated configuration with relative motion between rotor and struts. However, the focus of attention here is the rotor-strut interaction event, and it is hoped that FLUENT ${ }^{\circledR}$ can capture the large-structure flow field reasonably well. As a measure to check against possible loss of major acoustic mechanisms excluded by the current simulation, comparison with experiment is made for the time-domain pressure wave form. The specific questions asked in this study are as follows. (1) How important is the 3D flow effect in a design which seems to be based on two- 
TABLE I. Geometry of the rotor blades and the incidence angle distribution.

\begin{tabular}{llccc}
\hline \hline Span $(\%)$ & Chord $(\mathrm{m})$ & Stagger $(\mathrm{deg})$ & Camber $(\mathrm{deg})$ & Incidence $(\mathrm{deg})$ \\
\hline 0 & 0.0239 & 41.6 & 13.2 & 25.7 \\
18 & 0.0252 & 46.6 & 22.2 & 11.9 \\
35 & 0.0270 & 50.4 & 22.5 & 7.3 \\
53 & 0.0289 & 53.3 & 20.8 & 5.5 \\
71 & 0.0307 & 55.0 & 19.1 & 4.7 \\
89 & 0.0320 & 57.1 & 20.4 & 1.1 \\
100 & 0.0307 & 61.3 & 19.1 & -1.8 \\
\hline \hline
\end{tabular}

dimensional (2D) radial equilibrium? (2) What is the noise source distribution on the blade and strut surfaces, and how does noise from one part interfere acoustically with another? (3) What are the aerodynamic mechanisms of the surface pressure fluctuations which control the far-field noise radiation?

In what follows, Sec. II outlines the computational configuration and the calibration of the numerical scheme. The main results for the sound source are presented in Sec. III. Section IV is devoted to the comparison of the predicted sound and the acoustic measurement. Conclusions are drawn in Sec. V.

\section{COMPUTATIONAL MODEL AND MESH TEST}

\section{A. Physical fan model for experiment}

For simplicity, a free-delivery working condition is considered for both experimental study and numerical simulation although it is well known that the source mechanisms at other working conditions, e.g. near a surge point, can be very different. In order to focus on the rotor-strut interaction noise mechanism, a model fan was fabricated based physically on a real fan purchased from the market. The modifications essentially eliminated the noise radiated by the inlet flow distortion coming towards the rotor. The real fan is shown in Fig. 1(a). It is a typical 90-mm-diam cooling fan used in desktop computers. There are seven rotor blades rotating anti-clockwise when viewed from the front. There are four cylindrical struts of $4 \mathrm{~mm}$ in diameter, and the axial clearance between the cylinder and the blade trailing edge is $3 \mathrm{~mm}$. The design speed for the fan is $3000 \mathrm{rpm}$. The blade span is $25.5 \mathrm{~mm}$. The tip clearance is $1.5 \mathrm{~mm}$, or $6 \%$ of the span. The midspan chord length is $28 \mathrm{~mm}$. The main geometric features of the rotor blade are given in Table I. The struts lean towards the rotational direction and are almost tangent to the rotor hub within which the motor is located. The model fan had two modifications from the real fan shown in Fig. 1(a). The rationale for the modifications is described below. The inlet bellmouth shown in Fig. 1(a) is incomplete due to the square outer frame, and it has been shown to cause considerable extra noise (Huang and Wang, 2005). In order to focus attention on the rotor-strut interaction noise, a complete bellmouth with lip radius of $10 \mathrm{~mm}$ is used, as shown in Figs. 1(b) and 1(d), which have different view angles. The configuration of the four tangential struts in the real fan is highly three dimensional, and its noise pattern is a complex rotating dipole (Huang, 2003). To simplify the matter, seven radial struts are used instead. The back view of the struts is shown in Fig. 1(c). When the number of rotor blades coincides with the number of struts, the noise pattern is a simple thrust noise beaming along the rotational axis.

\section{B. Computational domain and boundary conditions}

Despite the recent advances in direct computation of flow-generated noise (Wang et al., 2006), acoustic analogy (Lighthill, 1952; Ffowcs Williams and Hawkings, 1969) remains as a reliable and efficient approach. As the free-space Green's function is used in the FW-H equation, the source should be calculated by compressible flow solvers with appropriate boundary conditions when the source is noncompact; otherwise a suitable Green's function should be used when pressure from incompressible flow solvers are used for low-speed flows (Howe, 1999). However, this requirement can be relaxed if the source is acoustically compact. In the present study, the Mach number is below 0.05 and the ratio of the fan diameter to the typical wavelength is less than 0.1 . Therefore, the incompressible flow solver is chosen together with a compact computational domain shown in Fig. 1(d). A cylindrical cross section view with mesh is given in Fig. 1(e). The model closely follows the physical model fan shown in Figs. 1(b) and 1(c), but there are minor modifications based on numerical needs and are expected to have minimal impact on computational results. The two minor modifications are explained as follows.

For the inlet, the only certainty is that the stagnation pressure is atmospheric and the velocity vanishes at a location very far away from the bellmouth. The actual volume flow rate of the rotor should be a result of numerical simulation with appropriate exit boundary conditions. The volume flow rate, hence the incoming flow velocity, cannot be determined a priori. Such prediction would pose a heavy demand on computational resources due to the large computational domain. In order to limit the demand on computational resources, there is a need to set an inlet computational domain boundary in the near field where the exact velocity is uncertain theoretically, but can be specified in practice if experimentally measured velocity data is available. In other words, the velocity is measured and not calculated. This is justified by the focus on the interaction dynamics of the rotor trailing edge with the struts, which are much further downstream. The exact location of such near-field inlet boundary is dictated by experimental convenience and is illustrated in Fig. 1(d) by vectors shown near the labels of "inlet flow." LaserDoppler Anemometry was utilized to measure the flow velocity on a plane $5 \mathrm{~mm}$ upstream of the frontier of the inlet bellmouth, which was also 1.4 times the axial chord length from the blade leading edge. Near the outer rim of the inlet bellmouth, velocity measurement was conducted over a cylindrical surface across which the inlet flow was most likely perpendicular to the fan axis and parallel to the local bellmouth curvature. The measured flow rate for the free delivery condition for this fan was $0.0302 \mathrm{~kg} / \mathrm{s}$, and the distribution of the inlet flow velocity vector is shown in Fig. 1(d). The average axial-flow velocity at the inlet was around $5 \mathrm{~m} / \mathrm{s}$ over the whole inlet plane. 
At the downstream end, the circular flow passage provided by the casing is flush with the trailing edge of the struts. Since the flow just downstream of the cylinder is nonuniform, it is impossible to specify any flow quantity which can be deduced either theoretically or determined experimentally. For convenience, the casing flow passage is further extended by about $10 \mathrm{~mm}$ from the rear edge of the struts. On this exit plane, the atmospheric static pressure is given for the outer radius of the exit plane, and the inner parts of the exit plane are required to satisfy a radial force equilibrium, which is one type of boundary condition provided by Fluent ${ }^{\circledR}$. Due to the swirling exit flow, the pressure near the center of the exit plane is lower than the atmospheric pressure. The boundary conditions elsewhere are straightforward. All structural surfaces are given the no-slip condition.

Since the rotor blades rotate while the struts remain stationary, the computational domain is divided into two parts separated by an artificial interface which is set midway between the blade trailing edge and the cylinder leading edge. All fluid properties are identical on the two sides of the virtual interface. The mesh is allowed to have relative sliding, and the continuity condition there is enforced by simple interpolation where nodes are not overlapped. The two computational domains are deliberately shown as being separated in Fig. 1(d) to illustrate the position of the computational interface. In Fig. 1(e), the interfaces are joined back together, and the $2 \mathrm{D}$ mesh on the $70 \%$ of the radial span is shown illustrating the relative positions of the rotor blades, which are shown as airfoils, and the downstream cylinders, which are shown as circles just downstream of the interface.

For the model of seven blades supported by seven struts, which is designated the "coincident" configuration (Wang and Huang, 2006), there are two ways the computation can be carried out. In the first, the whole rotor and the set of seven struts can be modeled without making any geometrical simplification. The second method is to calculate only one seventh of the flow passage through both rotor and struts in relative motion. In the second method, a periodic boundary condition is assumed in the circumferential direction. Such assumption would be invalid if unsteady flow mechanisms are not phase locked with the rotation. For example, vortex shedding from the cylindrical struts might be independent of the motion of the upstream rotor. In other words, there is a risk of losing some physical phenomena when only a single flow passage is calculated. The potential advantage, however, is that the mesh density can be much higher for the same amount of computing resources. Comparison of two computational configurations with the same mesh density shows that the single-passage calculation can be used.

\section{Numerical solver, mesh and convergence test}

The commercial software package of FLUENT ${ }^{\circledR}$ (version 6.3) was used to solve the unsteady, incompressible, Reynolds averaged Navier-Stokes equation. The Reynolds number based on the midspan rotor blade chord is 8700 , which is too low for any "natural" transition from laminar to turbulence, i.e., that arising from Tollmien-Schlichting wave development, to take place. However, factors like inlet flow turbulence or flow separation may cause early transitions. A "rule-of-thumb" critical Reynolds number would be 350000 (Mayle and Roberts, 1991). Therefore, the flow in the rotor region is unlikely to be turbulent although it is not impossible. The Reynolds number based on the strut diameter is 1590; therefore, the wake flow is likely to be turbulent (Zdravkovich, 1997). The cylinder is located downstream of the rotor and its inlet flow changes from a fairly uniform potential flow to the wake flow periodically. The lift and drag fluctuations induced by the changes in the incoming flow are shown to be much larger than those induced by its own vortex shedding which might well be turbulent; the data for the latter may be found from Norberg, 2001. Furthermore, numerical tests also showed that the timing of the vortex shedding from the cylinder was fully locked to the rotor-strut encounter, and the pattern of self-induced vortex shedding for an isolated cylinder no longer existed. The shedding frequency for an isolated cylinder is estimated as follows. For a cylinder of $4 \mathrm{~mm}$ in diameter, placed in a free cross stream of $6.4 \mathrm{~m} / \mathrm{s}$, which is the flow velocity relative to the strut, the Strouhal number for the vortex shedding frequency is around $\mathrm{St}=0.2$, hence $f=\mathrm{StU} / \mathrm{D}=0.2 \times 6.4 / 0.004=320 \mathrm{~Hz}$. This frequency is close to but below the blade passing frequency $(350 \mathrm{~Hz})$ of the rotor.

A first-order time-accurate scheme was used for the simulation, and the scaled convergence criterion for mass continuity was set at $10^{-4}$. For velocities in the axial and lateral directions, a scaled momentum conservation criterion was used and the limit was set at $10^{-6}$. For mass continuity, the scaled convergence is defined as the sum of the absolute mass imbalance over all cells divided by the largest such sum in the first five time steps. For the momentum conservation, the criterion is defined as the sum of the absolute momentum imbalance over all cells divided by the sum of absolute momentum fluxes across all cell boundaries. Tests with the second-order time-accurate scheme in FLUENT ${ }^{\circledR}$ showed that it was simply not robust enough for such a complex task.

Computations with three laminar/turbulence model settings were conducted for the rotor-strut interaction and the results are compared in Fig. 2(a). In the first, the laminar flow model is used in both the upstream rotor and the downstream strut domains. In the second, the flow in the rotor domain is assumed laminar, while that in the strut is turbulent. In the third model, both domains are assumed to be turbulent. For the turbulence model, the Reynolds stress model (RSM) is chosen together with the standard wall function treatment with $y^{+}=1$. Other turbulence models, such as $k$-epsilon and $k$-omega, were also tested but RSM was found to perform better in the sense discussed below. As a basic check on the impact of the choice of the flow models, threedimensional cross flow through an isolated strut is studied for the diameter based Reynolds number of 1600 . The mesh density near the cylinder surface is basically the same as that used in the rotor-strut interaction study, cf. Fig. 1(e). The mean drag, root-mean-square lift and the vortex shedding frequencies are given in Table II where they are also compared with experimental results from literature. It is found that both laminar solver and RSM give reasonable approximation for the shedding frequency and the mean drag, but 

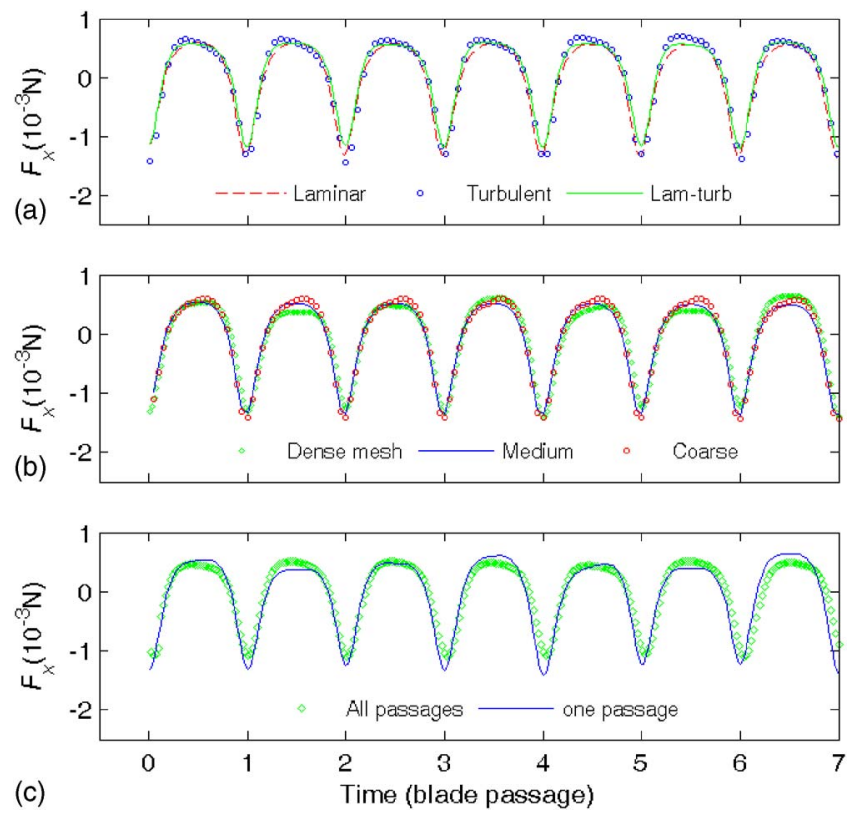

FIG. 2. Flow model and mesh test. (a) Total thrust $F_{x}$ calculated by three laminar/turbulent flow model settings for the rotor and strut domains. (b) Total thrust $F_{x}$ calculated by three levels of mesh density: dense mesh with $1.8 \times 10^{5}$ cells, medium mesh with $10^{5}$ cells and coarse mesh with 0.5 $\times 10^{5}$ cells. (c) Comparison of the single-passage calculation with the seven-passage calculation, both with high mesh density.

both overestimate the fluctuating lift. In this test, the RSM performs better than the laminar model, but the latter is also retained in this study to examine whether an overestimated wake fluctuation would have serious impact on the final sound radiation. No attempt is made to fine tune the turbulence model to match with the experimental data, which is well known to be a difficult task for certain ranges of Reynolds numbers (Cox et al., 1998; Norberg, 2001) and is beyond the scope of the present investigation. Coming back to the rotor-strut interaction, it is found that different models produce different details of pressure distributions over the strut surface as well as over the rotor pressure side. However, the pressure on the suction side of the rotor blade is relatively insensitive to the laminar/turbulent flow models adopted. The total axial force experienced by the rotor and strut, which is here defined as the thrust, $F_{x}$, and shown below to be crucial for the sound radiation, is given as a function of time in Fig. 2(a) for the three settings of flow models for the two domains. The difference of $F_{x}$ among the three predictions is minimal considering the differences found in the flow details, especially on the strut. It is observed that the force experienced by the strut is basically

TABLE II. Tests of laminar and turbulent flow models for 3D flow across an isolated strut at $R e_{d}=1600$.

\begin{tabular}{lccc}
\hline \hline Method $\rightarrow$ & Laminar flow & Reynolds stress & Experimental data \\
\hline $\begin{array}{l}\text { Strouhal } \\
\text { number }\end{array}$ & 0.19 & 0.19 & 0.21 (Norberg, 2001) \\
$\begin{array}{l}\text { Mean drag } \\
\text { coefficient }\end{array}$ & 1.1 & 0.94 & 1.0 (Zdravkovich, 1997) \\
$\begin{array}{l}\text { RSM lift } \\
\text { coefficient }\end{array}$ & 0.53 & 0.23 & 0.045 (Norberg, 2001) \\
\hline \hline
\end{tabular}

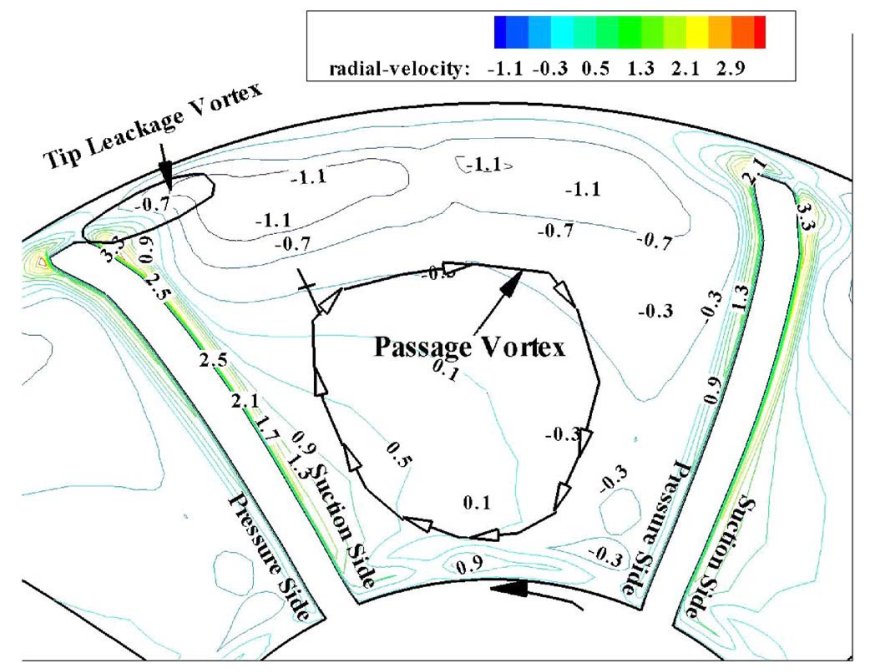

FIG. 3. Radial velocity contour in the rear region with the passage vortex and the tip leakage vortex illustrated.

cancelled out by that on the pressure side of the rotor blades, a phenomenon which is discussed in more detail in Fig. 5. Based on the earlier discussions on the likelihood of flow turbulence, the subsequent flow field analysis is based on the results obtained by the laminar flow for the rotor domain and the RSM for the strut domain.

Apart from these basic tests, tests using the singlepassage, laminar-flow model were also conducted to see what level of mesh density would be sufficient. The results are shown in Figs. 2(b) and 2(c). Figure 2(b) shows that the results for three mesh density levels, $5 \times 10^{4}$ cells (open circle), $1 \times 10^{5}$ cells (solid line) and $1.8 \times 10^{5}$ cells (diamond), are rather close. Note that the far-field sound is, in this case, essentially derived from the time derivative of the axial thrust force, $d F_{x} / d t$. Details are given in later sections. In addition to the mesh test, tests were also conducted to see if there was any difference between the single-passage model, for which a periodic boundary condition was imposed in the circumferential direction, and the complete model without imposing any circumferential boundary condition. The mesh density, or the number of cells per flow passage, used in the complete model was the same as that used in the single-passage flow model, the latter having $1.8 \times 10^{5}$ cells. The comparison is shown in Fig. 2(c). Given the moderate ratio of mesh cell counts, the convergence of results is satisfactory. In all these calculations, a total of at least 140 time steps were used for one rotational cycle for the unsteady flow computation.

\section{ANALYSIS OF THE INTERACTION MECHANISM}

\section{A. Features of three-dimensional flow}

Before analyzing the unsteady fluid dynamics of the rotor-strut interaction, the overall features of the threedimensional flow are studied by conducting a steady flow computation using the mixing-plane model at the interface between the rotor and struts. The steady flow field is shown in Fig. 3 for a cross section located at $85 \%$ of the axial blade chord from the plane of the leading edge. Despite the small 
size of the fan, the flow in the fan rotor is strongly three dimensional, mainly due to (a) the intrinsic fluid dynamics of boundary layers and, (b) large percentage of tip clearance. Shown in Fig. 3 is the contour of the radial velocity, superimposed by sketches of the tip leakage vortex and the passage vortex. The radial transport near the blade suction (convex) side, and the transport from the pressure (concave) side to the suction side on the hub, combined with the tip leakage flow from the suction side to the pressure side in the passage form the passage vortex. The tip leakage flow forms the leakage vortex in the midpassage region of the blade tip. Although the strut is installed in the radial direction and the blade span is also nearly radial, the rotor-strut interaction is a very three-dimensional phenomenon due to the 3D flow structure.

\section{B. Overall sound source distribution}

The interaction mechanism is first analyzed in terms of the overall sound source intensity distribution on the blade and strut surfaces. Here, the sound source is characterized essentially by the time derivative of the thrust force or the time derivative of the blade surface pressure, $\partial p / \partial \tau$. The analysis is then focused on the critical moments when the blade sweeps past the struts and strong lift fluctuation occurs. Other moments of weak interaction are also analyzed together with the overall phase angle relations for pressure fluctuations in all parts of the interacting surfaces.

Figure 4 shows the contours of $\partial p / \partial \tau$, with suction and pressure sides of the blades separated. Since the final radiated sound derives from a particular spectral component of $\partial p / \partial \tau$, the contour of the selected spectral component is also shown. Figures 4(a)-4(c) are contours of the root-meansquare values of $\partial p / \partial \tau$. Figure $4(\mathrm{c})$ is for strut surfaces in unwrapped view.

Locally, the sound source is found to be high in four regions.

(1) The pressure side surface near the trailing edge and $70 \%$ of the radial span.

(2) The corresponding trailing edge region on the suction side surface in a less concentrated manner.

(3) The midchord, blade tip region on the suction side where the leakage flow is strong.

(4) The region of strut surface between the front stagnation line and the expansion line, where alternating flow separation occurs.

The relation between the phase angle of the unsteady pressure on the two sides of the rotor blade is such that the sound radiated by the two sides basically adds to each other.

\section{Events surrounding the peak lift fluctuation}

Sound radiation is a result of acoustic interference among all surfaces. The phase relation between pressure fluctuations on the suction, pressure and strut surfaces is crucial for the final sound radiation. The relation is shown in Fig. 5(a) where the contributions to the integrated lift are decomposed for the time worth of two blade passages. For this particular example, the total thrust (simple solid line) is almost the same as the contribution from the blade suction surface (dashed line) since the contributions from the pressure side (solid line with downward triangle) are found to be almost completely cancelled out by that from the strut (solid line with open circles). It may be said that the pressure side of the blade is always "seen" by the strut and the two behave very much like two cylinders in tandem at a separation distance sufficiently large to allow this effect to cancel out. This observation is only true for the geometric parameters chosen; changing them could alter the results. Notice, however, that the cancellation may not be so complete when the working parameters are changed.

Figure 5(b) shows the time derivative of the total thrust. Three moments, labeled as A, B and C, are identified for further analysis of the flow field. The pressure contours for the three moments are shown in Fig. 6 for the revolution surface on the $70 \%$ of the radial span. The event near the peak, labeled as A, occurs when the center of the strut is immediately downstream of the blade trailing edge. Figure 6(a) shows that, due to the relative motion, the flow towards the cylinder hits the upper left side of the cylinder, where the stagnation point is located. The high pressure here essentially blocks the flow passing over the suction side of the blade, leading to a sudden increase of pressure on the suction surface. The opposite event occurs for the pressure side of the blade. The expansion point on the lower-right quadrant provides a low pressure condition for the flow over the pressure side leading to a decrease of pressure there compared with if the stagnation point were on the lower left without rotor-strut relative motion.

An interesting feature is found for times B and C and, to a lesser extent, also for time A. For the pressure side of the blade, pressure contour lines intercept the surface curve, meaning that the pressure increases from the leading edge towards the trailing edge. On the suction surface, however, the contour lines are almost parallel with the surface. In other words, the pressure response on the upper surface is almost uniform.

\section{Weak interactions}

During much of the time period from A to C in Fig. 5(b), the total thrust force is almost constant, cf. solid line in Fig. 5(a). In this period, the strut is passing through the "potential core" region of the blade passage and the blade-strut interaction is weak. The pressure contours of $\mathrm{B}$ and $\mathrm{C}$ are shown in Figs. 6(b) and 6(c). Two observations are made.

(a) As time advances from $\mathrm{B}$ to $\mathrm{C}$, the pressures on the pressure side of the blade and on the strut change in a smooth manner, and the two almost cancel each other out in terms of their contributions towards the integrated thrust. However, the suction surface of the blade maintains almost constant pressure, as shown in Figs. 5(b) and 5(c). This could be a result of a shielding effect of the suction surface from the downstream cylinder. In other words, the cylinder can only be seen by the pressure side of the blade during this period of time.

(b) Moving from time B to time C, again as shown in Figs. $5(\mathrm{~b})$ and 5(c), the front stagnation point of the cylinder 

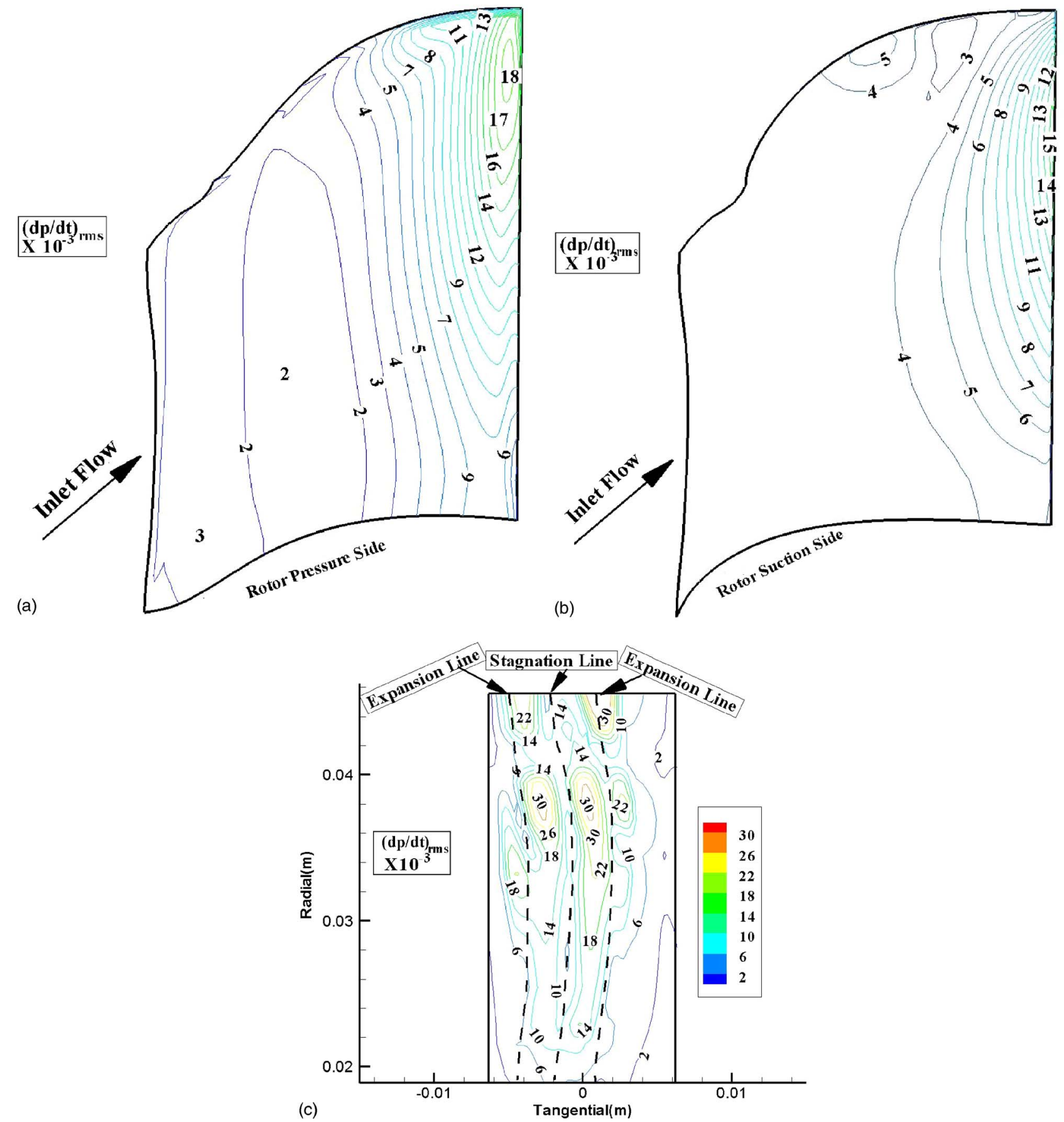

FIG. 4. Contours of the sound source term $\partial p /\left.\partial \tau\right|_{r m s}$ on (a) the rotor pressure side, (b) the rotor suction side, and (c) the strut suction and pressure sides in unwrapped view.

projects a "column" of high pressure region which is connected to the trailing edge of the blade, leading to a maximum pressure on the pressure side of the blade. Since the thrust force $F_{x}$ described here is the force acting on the fluid from the blade, high pressure on the blade pressure side means a high thrust in the positive $x$ direction. For the strut, the thrust acting on the fluid is directed towards upstream, hence the negative value.

\section{PREDICTION OF SOUND AND EXPERIMENTAL VALIDATION}

It is difficult to measure the unsteady flow field experimentally on the rotating blades. The comparison between the computation described above and the experiment is conducted at the acoustic level. The sound radiation predicted by integrating the Ffowcs Williams-Hawkings' acoustic equation is compared with the measured sound in terms of (a) the acoustic directivity of sound intensity, and (b) the timedomain sound pressure signature.

\section{A. Computation of the radiated sound}

The prediction of the radiated sound follows the well established Ffowcs Williams-Hawkings' equation (1969), which is the extension of the Lighthill acoustic analogy. With 

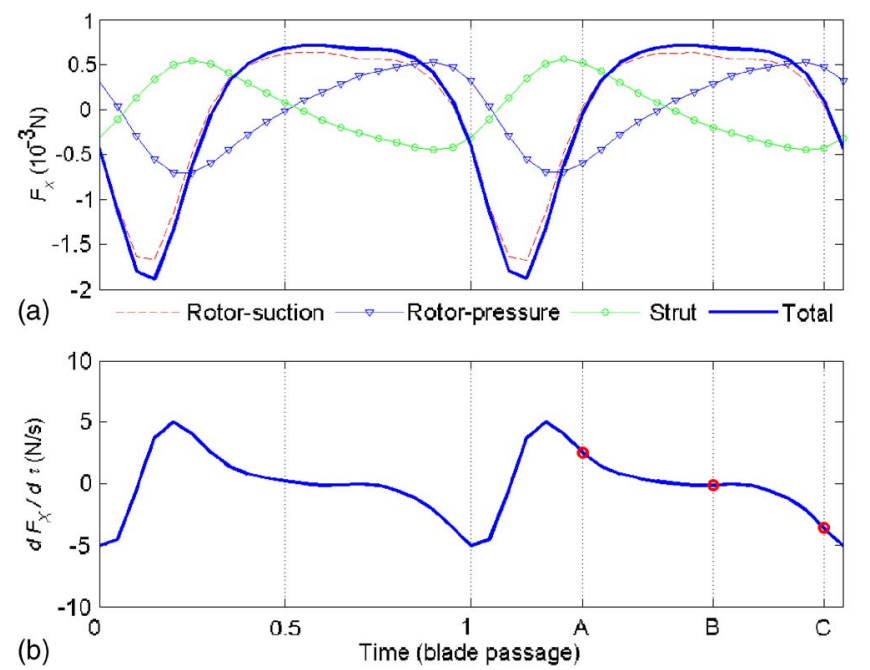

FIG. 5. Strong and weak interaction periods. (a) Decomposition of the thrust forces into contributions from the two blade surfaces and the strut. (b) The time derivative of the total thrust.

the quadrupole term neglected for the low-speed applications, the equation is written in its integral form as

$$
\begin{aligned}
p(\mathbf{x}, t)= & \frac{\partial}{\partial t} \int_{S}\left[\frac{\rho_{0} v_{n}}{4 \pi r\left|1-M_{r}\right|}\right]_{\mathrm{ret}} d S \\
& -\frac{\partial}{\partial x_{i}} \int_{S}\left[\frac{F_{i}}{4 \pi r\left|1-M_{r}\right|}\right]_{\mathrm{ret}} d S,
\end{aligned}
$$

where $x=\left(x_{1}, x_{2}, x_{3}\right)$ is the position of the observer, $t$ is the reception time, $\rho_{0}$ is the undisturbed fluid density, $c_{0}$ is the speed of sound, $S$ is the source (blade) surface which moves at an outward normal velocity of $v_{n}, r=|x-y|$ is the sourceto-observer distance, $M_{r}$ is the Mach number of the source movement velocity projected to the $r$ direction, $F_{i}$ is the pressure force acting on the surface projected to the $x_{i}$ direction, and subscript "ret" implies evaluation at the retarded time

$$
\tau=t-\frac{r}{c_{0}}
$$

when the sound is radiated. Note that the square brackets in Eq. (1) are evaluated at this retarded time. The actual implementation of Eq. (1) is easier if the time and space derivatives are absorbed into the integrands which depend on $x_{i}$ and $t$ through the retarded time $\tau$ and the factor of $r$. The result is the so-called Formulation 1A derived by Farassat and Succi (1983)

$$
\begin{aligned}
p=p_{T}^{\prime} & +p_{L}^{\prime}, \\
4 \pi p_{T}^{\prime}= & \rho_{0} \int_{S}\left[\frac{\left(\dot{v}_{n}+v_{\dot{n}}\right)}{r\left|1-M_{r}\right|^{2}}\right. \\
& \left.+\frac{v_{n}\left(r \dot{M}_{r}+c_{0}\left(M_{r}-M^{2}\right)\right)}{r^{2}\left|1-M_{r}\right|^{3}}\right]_{\mathrm{ret}} d S,
\end{aligned}
$$

$$
\begin{aligned}
4 \pi p_{L}^{\prime}= & \frac{1}{c_{0}} \int_{S}\left[\frac{\dot{F}_{r}}{r\left|1-M_{r}\right|^{2}}+\frac{c_{0}\left(F_{r}-F_{M} M\right)}{r^{2}\left|1-M_{r}\right|^{2}}\right. \\
& \left.+\frac{F_{r}\left(r \dot{M}_{r}+c_{0}\left(M_{r}-M^{2}\right)\right)}{r^{2}\left|1-M_{r}\right|^{3}}\right]_{\mathrm{ret}} d S,
\end{aligned}
$$

where the sound pressure is divided into thickness (subscript $T$ ) and loading (subscript $L$ ) contributions, the dot over variables is the retarded time derivative, $F_{M}$ is the force projected to the source movement direction. For the current problem, the time derivative for the Mach number is essentially the centripetal acceleration

$$
\dot{M}_{r}=\frac{\partial \mathbf{M}}{\partial \tau} \cdot \frac{\mathbf{r}}{r}=-\omega M \frac{\mathbf{r}_{s} \cdot \mathbf{r}}{r_{s} r}, \quad \frac{\mathbf{r}_{s} \cdot \mathbf{r}}{r_{s} r}=\sin \alpha
$$

where $r_{s}$ is the cylindrical radius of the source element $d S$ with respect to the rotational axis, and $\alpha$ is the latitudinal angle of the observer measured from the rotational axis.

Notice that the terms with a distance dependence of $r^{-1}$ is the far-field sound, while those with $r^{-2}$ are the near-field sound. Since the computed sound is to be compared with experimental measurement at a distance of $r_{0}=0.5 \mathrm{~m}$ from the fan center, and the wavelength of the blade passing frequency is $c_{0} / f=340 / 350=0.97 \mathrm{~m}$, there is a certain nearfield effect. The effect of the near field on the measured sound pressure signature is discussed later.

In the third group of the third expression of Eq. (3), there are both far-field and near-field terms, the one associated with $F_{r} \dot{M}_{r}$ being the far-field term. This term joins the first term of $\dot{F}_{r}$ and form the far-field loading noise

$$
p_{L \infty}^{\prime}(\mathbf{x}, t)=\frac{1}{4 \pi c_{0}} \int_{S}\left[\frac{\dot{F}_{r}}{r\left(1-M_{r}\right)}-\frac{F_{r} \omega M \sin \alpha}{r\left|1-M_{r}\right|^{3}}\right]_{\mathrm{ret}} d S .
$$

When the loading measured in the rotating reference frame is a constant, $F_{r}(\tau)=$ const, the second term in the above equation makes far-field noise by the changing radiation distance due to the source movement. This is part of the so-called Gutin noise, which also includes the far-field terms of $p_{T}^{\prime}$ in Eq. (3). Gutin noise is only significant when the rotating speed is reasonably high. Using the formulations of (Lowson, 1970) and the approximation for small tip Mach number, $M=0.037$ in this case, it can be shown that the ratio of the sound pressure radiated by the Gutin noise to that of the interaction noise is proportional to $L_{0}(B M)^{B} / L_{1}$, where $L_{0}$ is the amplitude of the steady flow loading, $L_{1}$ is the amplitude of the blade passing frequency (BPF) component of the loading caused by the interaction, and $B$ is the number of blades, seven in this case. A detailed estimate for the present fan working in free delivery condition indicates that the integrated sound power from the Gutin noise is about $70 \mathrm{~dB}$ below that of the interaction noise. Back to Eq. (5), the rotorstator interaction noise derives from the unsteady loading $\dot{F}_{r}$. The dominant far-field noise in this case is approximated by neglecting the factor of $1-M_{r}$ in Eq. (5) and the Gutin noise 


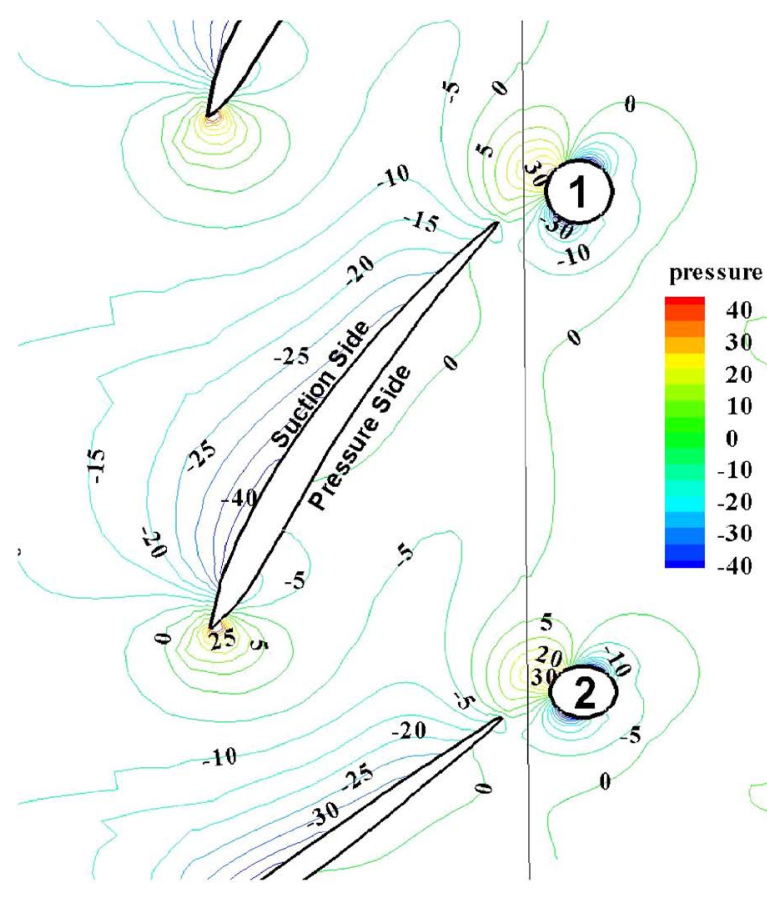

(a)
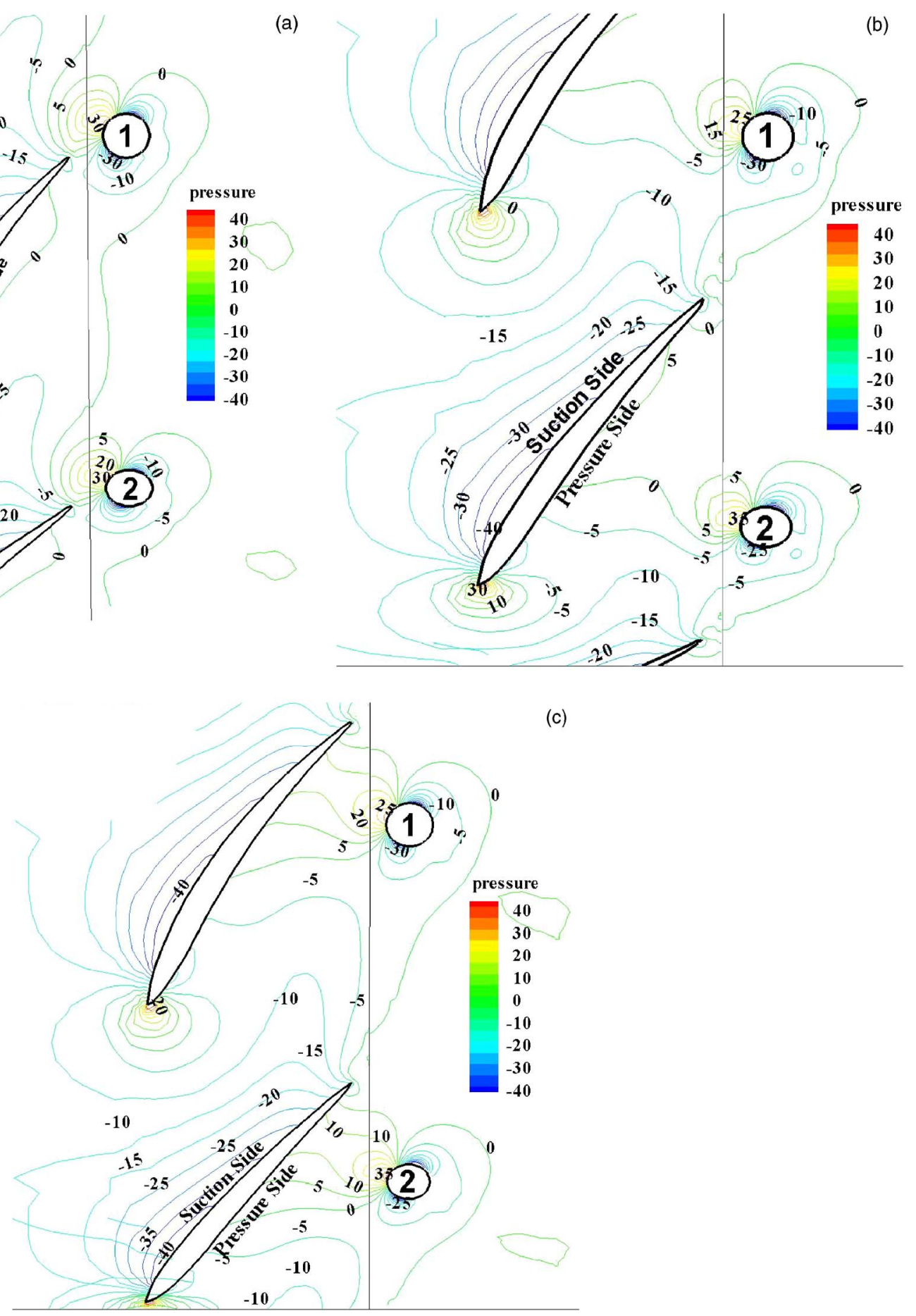

(c)

FIG. 6. The pressure contours on the revolution surface of 70\% radial span at three moments A, B, and C marked in Fig. 5(b).

$$
p_{\infty}(\mathbf{x}, t) \approx\left[\frac{\partial}{\partial \tau} \int_{S} F_{r} d S\right]_{\mathrm{ret}} /\left(4 \pi r_{0} c_{0}\right),
$$

where $r_{0}$ is the distance of the observer to the center of the fan. Also, when the forces do change with time, the second term of Eq. (5) should be computed as part of the non-Gutin noise. Besides, all loading noise terms associated with $F_{r}$ and $F_{M}$ in Eq. (3) should also be computed for the purpose of comparison with the experimentally measured sound in the near field.

Equation (6) is a point-source approximation for small and slow axial-flow fans, and its deviation from the full integration of Eq. (5) represents a measure of source noncompactness, which is apparently small for the present study. Having said that, it does not mean that there is no acoustic interference between sound waves radiated at different parts of the fan. The interference arises mainly through different phase angle distributions on the blades, which determines the outcome of $\left[\int_{S} F_{r} d S\right]_{\text {ret }}$ in Eq. (6). As shown earlier, the suction surface of the rotor blade seems to have rather uniform phase angle, as evidenced by the pressure contours parallel to the surface in Fig. 6, but the pressure surface has a diverse 


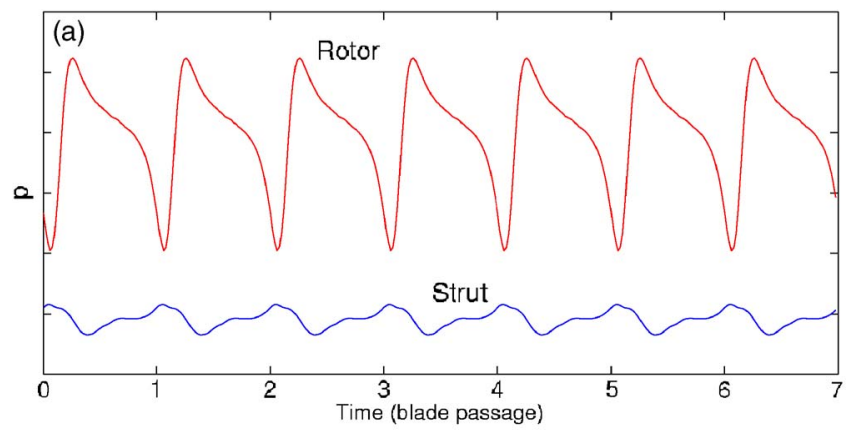

(b)
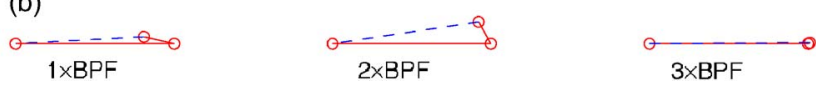

FIG. 7. Rotor-strut acoustic interference. (a) Sound pressure decomposition into rotor (upper curve) and strut (lower curve) contributions. (b) Vectorial representation of complex sound amplitudes for the first three blade passing frequencies. The broken lines are the total sound amplitude consisting of the rotor (horizontal line) and strut (oblique line) contributions.

phase distribution. Nevertheless, it is physically more straightforward to study the acoustic interference between the sound waves radiated by the rotor blades and those by the struts, instead of waves from the two surfaces of the rotor blades. The result of the rotor-strut acoustic interference is shown in Fig. 7 for sound received at $r_{0}=0.5 \mathrm{~m}$ upstream of the fan center along the rotational axis.

Figure 7(a) decomposes the sound radiated by the rotor blades (upper curve) and struts (lower curve) in a waterfall format with the same vertical scale. Overall, the ratio of the linear pressure amplitudes is 5.8 in favor of the rotor blades. Considering the fact that the strut is downstream of the rotor, this result reveals that the aeroacoustic mechanism in a small fan like this differs from the familiar interaction mechanism between a rotor and profiled stator blades. In the current configuration, the rotor experiences periodic change in the blockage by the downstream strut. This is mainly a potential flow interaction. The struts, on the other hand, experience drastic changes in the incoming flow due to the rotor wakes periodically hitting the struts. The interaction has both viscous and inviscid contributions. Due to the nature of the bluff body, the oscillating force occurring on the strut is not as impulsive as that which occurs on the rotor blades.

Figure 7(b) analyzes the acoustic interference in terms of the first three BPF harmonics. First, Fourier transform is applied to both sound waves shown in Fig. 7(a). The complex amplitude of the strut sound is then divided by that of the rotor sound. The complex ratio is shown in vector form. The horizontal line has a length of unity and it represents the rotor noise. The connecting oblique solid line represents the strut noise and the broken line is the total noise. The phase angle differences between the rotor and strut sounds are $167.1^{\circ}, 119.5^{\circ}$, and $26.2^{\circ}$, for the $(1-3)$ BPF frequencies, respectively. The length of the strut noise is seen to decrease with the harmonic index of the BPF. In other words, the strut noise features more at low frequencies. The interference is destructive for the first two frequency components. The strut noise for the third BPF is insignificant, so is its interference with the rotor noise. If the strut is considered to produce

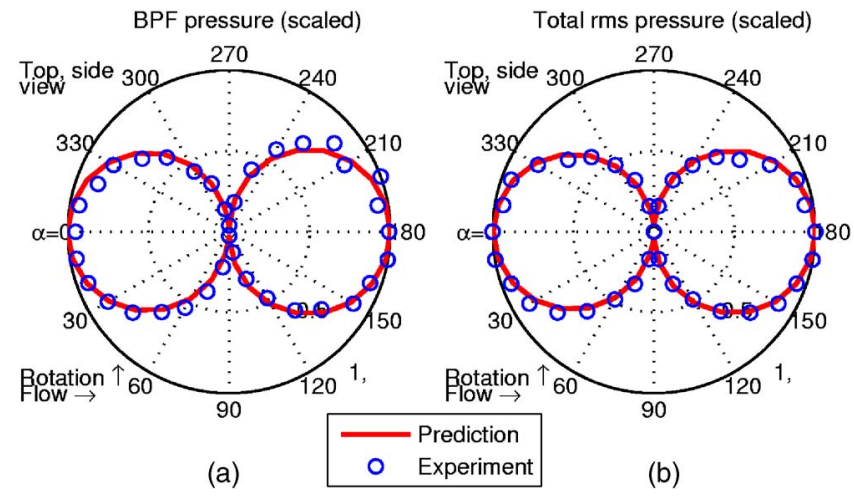

FIG. 8. Comparison of the acoustic directivity of sound pressure (rms) between experiment $(O)$ and prediction $(-)$ for the (a) BPF and (b) all frequencies. Both are scaled by the values on the pressure on the rotational axis. The directivity is taken on the horizontal plane while the fan stands in its upright position with flow from left to right.

cancellation noise, its timing is found to lag behind the rotor by $12.9^{\circ}, 60.5^{\circ}$ for the first two BPFs, respectively.

\section{B. Experiment and comparison}

Experimentally, it is only possible to measure the total sound radiated by the fan and this is compared with the prediction as follows. The acoustic measurement was conducted in the full anechoic chamber. Details of the general setup are given in (Huang and Wang, 2005). The measurement microphone was placed at $r_{0}=0.5 \mathrm{~m}$ from the fan center. The reason why the measurement was not taken at a much longer distance from the fan center was that higher frequency components became, as shown in Fig. 9(b), rather faint for the microphone to capture, higher frequency component being crucial for the detailed wave signature during the event of rapid rotor-strut encounter. The sampling rate is $16 \mathrm{kHz}$, and the BPF is $350 \mathrm{~Hz}$. A special feature in this measurement is that sound waves are averaged over many rotational cycles determined by the tachometer signals. The time period for the rotational cycle varies as the fan speed varies from one cycle to the next. Typically, the rpm varies by \pm 10 with a mean of 3000 in one test lasting $10 \mathrm{~s}$. This variation is corrected and the synchronous average of the sound signal thus derived has a sharp peak on the BPF and its higher order harmonics. Without such time-base correction, spectral leak or broadening would occur.

The comparison of acoustic directivity is shown in Figs. 8(a) and 8(b) for the BPF component and the total rotary noise, respectively. Experimental results are shown in open circles and the numerical predictions in solid curves. In order to focus on the distribution pattern, both experimental results and the predictions are normalized by the rms sound pressure on the rotational axis. The orientation of the fan is such that the flow goes from $0^{\circ}$ to $180^{\circ}$ (left to right), and the axis formed between $90^{\circ}$ and $270^{\circ}$ represents the rotational plane. Very close agreement is found in terms of the spatial distribution. The pattern is a clear thrust dipole (Huang, 2003) as no drag noise can be radiated in this coincident design in which the number of rotor blades is equal to the number of struts. When sound intensity is integrated over the measurement sphere, sound power is obtained. For the BPF, the pre- 

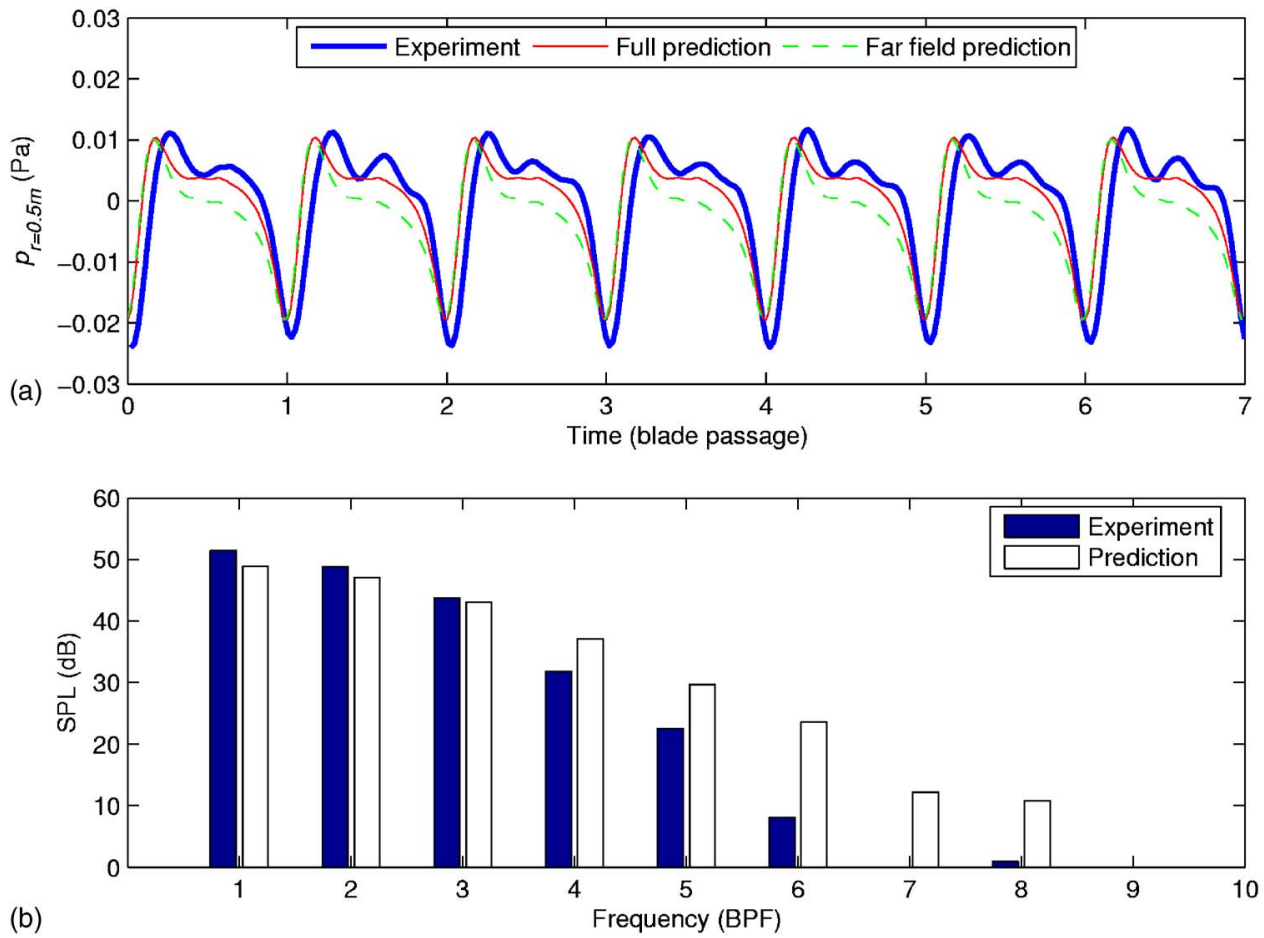

FIG. 9. Comparison of experimental measurement and numerical prediction of sound at $0.5 \mathrm{~m}$ upstream of the fan center on the rotational axis. (a) Pressure wave forms. (b) Spectra. diction is $48.9 \mathrm{~dB}$ (re $10^{-12} \mathrm{~W}$ ) while the experiment is $51.7 \mathrm{~dB}$. When all BPF harmonics are added, the prediction has $51.8 \mathrm{~dB}$ while the experiment has $53.8 \mathrm{~dB}$. The noise is under predicted by about 2.8 and $2.0 \mathrm{~dB}$ for the BPF and all frequencies, respectively.

Since the radiation of sound peaks on the rotational axis, the comparison of prediction and experiment is conducted for the upstream axial point. The result is shown in Fig. 9. Figure 9(a) shows that there is a general agreement of wave form between the measured sound pressure (thick solid line) and the predicted one (thin solid line). Both show a rather sharp impulse for each blade passage in front of struts, but there are several differences. First, the falling curve and the rising edge of the impulse are rather symmetrical with respect to the trough in the experimental data while the computational results are not. Second, the measured sound pressure features some ringing ripples, while the computation gives a smooth curve in the region between two consecutive impulses. Having said this, the overall signatures agree with each other very closely.

When the near-field terms are neglected in the prediction, the radiated sound is given by Eq. (5) and the result is shown as the dashed line in Fig. 9(a). The major difference between the near-field sound (thin solid line) and the far-field sound (dashed line) lies in the pressure level during the weak-interaction period illustrated by Figs. 6(b) and 6(c). The near-field effect maintains the pressure at a high level following the strong interaction, while the far-field radiation features more symmetrical impulses before and after the rotor-strut encounter.

Figure 9(b) compares the spectra of the experiment (filled bar) and the near-field prediction (open bar). The sound pressure level for the fundamental blade passing frequency $(\mathrm{BPF})$ is very close, the prediction being $2.5 \mathrm{~dB}$ lower than the experiment. Over prediction occurs when the order of harmonics is greater than 3. These over predictions are obvious manifestations of the sharp rise of sound pressure following the rotor-strut encounter. The discrepancy of the total sound pressure level is mainly controlled by the first few peaks, and the prediction is $1.9 \mathrm{~dB}$ below the experiment. This difference could be attributed to many factors: such as the difference between the real flow conditions and the boundary conditions imposed in the numerical simulation for the sake of numerical convenience, possible inlet flow distortions occurring in the experiment.

\section{CONCLUSIONS}

This study is motivated by the understanding of the aeroacoustic mechanism of the tonal noise radiated by a well-designed fan. The study is based on the numerical simulation of incompressible flow through a rotor and a set of downstream struts. The physical construction of the fan is such that a two-dimensional aerodynamic design procedure might suffice as both the blades and the struts are almost aligned in the radial direction. However, the flow field is found to be highly three-dimensional due to the existence of strong tip leakage flow, passage vortex, and radial transport of boundary layer flow, or the secondary flow. Threedimensional, unsteady flow is simulated by FLUENT ${ }^{\circledR} 6.3$ for which the convergence test for mesh density and benchmark tests are conducted. The simulated thrust fluctuation is compared with the acoustic measurement, and the overall discrepancy in terms of the total sound power is around $2.0 \mathrm{~dB}$. The numerical results are analyzed to reveal a few interesting phenomena of the rotor-strut interaction. The following conclusions are drawn.

(1) When compared with the acoustic measurement, the 3D simulation method adopted in the current study seems to under predict the lift deficit and recovery immediately 
following the passing of the blade trailing edge in front of the strut. Nevertheless, it is argued that the main character of the thrust fluctuations is captured satisfactorily.

(2) The fluctuating forces are derived from contributions from three parts: the blade suction (convex) surface, the blade pressure (concave) surface, and the strut. The two latter factors seem to cancel each other out fairly completely, and the overall signature resembles closely the response of the suction surface.

(3) The suction surface experiences sudden pressure increase, hence lift deficit, when the cylinder (strut) first emerges from the pressure side of the flow passage. The response on the whole chord length is rather uniform, unlike the pressure side response which is more concentrated near the trailing edge. In terms of the radial distribution of the sound source, highest response is found at around $70 \%$ of the blade span.

(4) The mechanism of the suction surface pressure increase is explained by the blockage of the flow past the suction surface when the front stagnation point provides a high back pressure for the suction surface.

(5) The sound radiated by the coincident design of $B=S$ features thrust dipole along the rotational axis. The acoustic interference between the rotor and the strut is destructive and the strut sound lags behind the rotor sound.

\section{ACKNOWLEDGMENT}

The project is supported by the Central Allocation Scheme of the Research Grants Council of the Hong Kong SAR Government (PolyU1/02C).

Blake, W. K. (1986). Mechanics of Flow-Induced Sound and Vibration (Academic, Orlando, FL).

Chiu, W. S., Lauchle, G. C., and Thompson, D. E. (1989). "Subsonic axialflow fan noise and unsteady rotor force," J. Acoust. Soc. Am. 85, 641647.

Cox, J. S., Brentner, K. S., and Rumsey, C. L. (1998). "Computation of vortex shedding and radiated sound for a circular cylinder: Subcritical to transcritical Reynolds numbers," Theor. Comput. Fluid Dyn. 12, 233-253.

Cudina, M. (1992). "Noise generated by a vane-axial fan with inlet guide vanes," Noise Control Eng. J. 39, 21-30.

Farassat, F., and Succi, G. P. (1983). "The prediction of helicopter discrete frequency noise," Vertica 7, 309-320.

Ffowcs Williams, J. E., and Hawkings, D. L. (1969). "Sound generation by turbulence and surfaces in arbitrary motion," Philos. Trans. R. Soc. London, Ser. A 264, 321-342.

Fukano, T., Takamatsu, Y., and Kodama, Y. (1986). "The effects of tip clearance on the noise of low pressure axial and mixed flow fans," J.
Sound Vib. 105, 291-308.

Kameier, F., and Neise, W. (1997). "Rotating blade flow instability as a source of noise in axial turbomachines," J. Sound Vib. 203, 833-853.

Kemp, N. H., and Sears, W. R. (1953). "Aerodynamic interference between moving blade rows," J. Aeronaut. Sci. 20, 583-598.

Kemp, N. H., and Sears, W. R. (1955). "Unsteady forces due to viscous wakes in turbomachines," J. Aeronaut. Sci. 22, 478-483.

Hodson, H. P. (1985). "Measurements of wake-generated unsteadiness in the rotor passages of axial flow turbines," J. Eng. Gas Turbines Power 107, 467-476.

Howe, M. S. (1999). "Trailing edge noise at low Mach numbers," J. Sound Vib. 225, 211-238.

Huang, L. (2003). "Characterizing computer cooling fan noise," J. Acoust. Soc. Am. 114, 3189-3200.

Huang, L., and Wang, J. (2005). "Acoustic analysis of a computer cooling fan,” J. Acoust. Soc. Am. 118, 2190-2200.

Huang, L., Zou, Z. P., and Xu, L. (2003). "Prediction of computer cooling fan noise using a 3D unsteady flow solver," Noise-Con 2003, Cleveland, Ohio, 23-25 June.

Lighthill, M. J. (1952). "On sound generated aerodynamically: I. General theory,” Proc. R. Soc. London, Ser. A 211, 564-587.

Lowson, M. V. (1965). "The sound field for singularities in motion," Proc. R. Soc. London, Ser. A 286, 559-572.

Lowson, M. V. (1970) "Theoretical analysis of compressor noise," J. Acoust. Soc. Am. 47, 371-385.

Mailach, R., Muller, L., and Vogeler, K. (2003). "Experimental investigation of unsteady force on rotor and stator blades of an axial compressor," Proc. Fifth Euro. Conf. Turb.-Fluid Dyn. Thermo. pp. 221-223, M. Stastny, C. H. Sieverding, and G. Bois, editors., March 18-21, Prague, Czech Republic.

Mailach, R., and Vogeler, K. (2004a). "Aerodynamic blade row interactions in an axial compressor-Part 1: Unsteady boundary layer development," J. Turbo. 126, 35-44.

Mailach, R., and Vogeler, K. (2004b). "Aerodynamic blade row interactions in an axial compressor-Part 2: Unsteady profile pressure distribution and blade force," J. Turbo. 126, 45-51.

Majumdar, S. J., and Peake, N. (1998). "Noise generation by the interaction between ingested turbulence and a rotating fan," J. Fluid Mech. 359, 181216.

Mayle, R. E., and Roberts, W. B. (1991). "The role of laminar-turbulent transition in gas turbine engines," ASME J. Turbomach. 113, 509-537.

Mayle, R. E., and Dullenkopf, K. (1989). "A theory for wake-induced transition," J. Turbo. 111, 188-195.

Norberg, C. (2001). "Flow around a circular cylinder: Aspects of fluctuating lift,” J. Fluids Struct. 15, 459-469.

Trunzo, R., Lakshminarayana, B., and Thompson, D. E. (1981). "Nature of inlet turbulence and strut flow disturbances and their effect on turbomachinery rotor noise," J. Sound Vib. 76, 233-259.

Wang, J., and Huang, L. (2006). "Quantification and control of noise sources in a small axial-flow fan," Noise Control Eng. J. 54, 27-32.

Wang, M., Freund, J. B., and Lele, S. K. (2006). "Computational prediction of flow-generated sound," Annu. Rev. Fluid Mech. 38, 483-512.

Washburn, K. B., and Lauchle G. C., (1998) "Inlet flow condition and tonal sound radiation from a subsonic Fan," Noise Control Eng. J. 31, 101-110. Zdravkovich, M. M. (1997). Flow Around Circular Cylinders Vol. 1: Fundamentals (Oxford University Press, Oxford). 